\title{
EchoGéo
}

$56 \mid 2021$

Les dynamiques spatiales contemporaines de la

Russie

\section{Points de vue et créations paysagères. Être un paysan agroforestier}

Olivier Bories, Jean-Pascal Fontorbes et Anne-Marie Granié

\section{(2) OpenEdition}

Journals

Édition électronique

URL : https://journals.openedition.org/echogeo/21668

DOI : 10.4000/echogeo. 21668

ISSN : 1963-1197

Éditeur

Pôle de recherche pour l'organisation et la diffusion de l'information géographique (CNRS UMR 8586)

Référence électronique

Olivier Bories, Jean-Pascal Fontorbes et Anne-Marie Granié, «Points de vue et créations paysagères. Être un paysan agroforestier», EchoGéo [En ligne], 56 | 2021, mis en ligne le 25 juin 2021, consulté le 31 juillet 2021. URL : http://journals.openedition.org/echogeo/21668 ; DOI : https://doi.org/10.4000/ echogeo. 21668

Ce document a été généré automatiquement le 31 juillet 2021.

EchoGéo est mis à disposition selon les termes de la licence Creative Commons Attribution - Pas d'Utilisation Commerciale - Pas de Modification 4.0 International (CC BY-NC-ND) 


\title{
Points de vue et créations paysagères. Être un paysan agroforestier
}

\author{
Olivier Bories, Jean-Pascal Fontorbes et Anne-Marie Granié
}

\section{« Je suis paysan agroforestier »}

1 C'est un paysage de campagne qui accroche l'œil, qui surprend au détour d'une itinérance, niché discrètement dans les plis arasés des grands pourtours de l'agglomération toulousaine. Un paysage inhabituel pour lequel on s'arrête au bord de la route, que l'on photographie ou que l'on dessine sur son carnet de terrain, comme nous l'avons fait. Une rareté dans le territoire que l'on consigne en images pour en garder un souvenir, une trace, et la montrer à d'autres. Une gaieté dans des paysages abimés par des campagnes d'arrachages. Une heureuse surprise qui interroge celui ou celle qui la débusque, un peu par hasard. C'est un paysage en files d'arbres qui s'allongent dans les champs, les prairies et qui détourent en haies leurs rebords. C'est un paysage agroforestier, une association d'arbres et de cultures ou d'animaux dans ou aux abords d'une même parcelle (MAAF, 2019). Des bouts de bocage reconstruits, ça et là, pas bien grands, quelques hectares à peine, qui redonnent une dimension à l'immensité de ces terres agricoles et qui les recomposent par endroits. Ils ne s'y diluent pas, au contraire, ils y existent et reprennent une taille, humaine. Ce sont les paysages agroforestiers que nous avons étudiés dans notre recherche en essayant de comprendre ce qu'ils font ici, pourquoi et comment? Nous l'avons fait à travers l'observation et la description de la figure agroforestière d'une part, et d'autre part à travers les hommes qui la fabriquent. Ces hommes sont "paysans agroforestiers». Nous ne prétendons pas qu'ils sont tous paysans, mais c'est bien comme cela que se nomment ceux que nous avons rencontrés ${ }^{1}$. Nous savions ${ }^{2}$ déjà que leurs profils, modelés par des parcours de vie, des découvertes et des expériences (de formation, de voyage, de pratiques agroécologiques...) provoquent une curiosité qui appelle chez eux 
une attention pour le paysage (Bories et Rue, 2017), une conscience paysagère (Bories, 2008).

2 Ainsi nous avons fait l'hypothèse dans la recherche que le paysage agroforestier n'est pas seulement le résultat dans le territoire d'une quête de performance : agronomique, écologique, économique... comme l'indiquent toutes les études sur le sujet et les encouragements à planter portés par des organismes techniques et de recherche. Le paysage agroforestier n'est pas seulement l'image d'un bénéfice et l'occasion d'un gain compté et comptabilisable, désincarné, mais il est aussi l'opportunité d'un reflet. Nous avons voulu vérifier que le paysage agroforestier agit comme un miroir dans le territoire de ce que sont ces paysans agroforestiers, en tant qu'Hommes, des valeurs qu'ils portent, de leurs engagements et de leurs convictions à faire et à montrer une autre agriculture. Nous avons supposé que le paysage agroforestier pût être une mise en image de leurs sensibilités, une projection incarnée d'eux-mêmes dans le lieu, comme un accord de complicité passé avec la terre qu'ils travaillent. Ceci nous a permis de mieux comprendre et d'analyser ce qui unit l'Homme, ici agroforestier, à son action sur le territoire. Ainsi nous sommes rentrés dans l'intimité de ces paysans qui plantent des arbres dans leurs parcelles et par l'action agroforestière nous sommes rentrés dans le sens du vivre avec l'autour. Nous avons conduit une recherche sociogéographique et paysagère qui articule un questionnement sur les trajectoires de vie, les représentations et un questionnement sur le paysage comme une restitution " esthétique " dans le cadre du pays, d'une façon d'être au monde, de s'y situer, de le pratiquer, d'agir sur lui, de l'habiter, d'en prendre soin... Nous avons fait le choix de filmer tout cela, de mener et d'écrire notre recherche avec les images et les sons, de mettre à l'écran le paysage agroforestier et les émotions de ceux qui le fabriquent ${ }^{3}$, de « faire du paysage un personnage » (Bories, 2019).

3 Nous étions convaincus d'une correspondance sensible entre le sujet paysager et l'écriture filmique pour fixer, enregistrer, restituer et discuter de nos résultats, dans une continuité de sens avec nos cadres théoriques, que sont la géographie des émotions et la sociologie compréhensive (Guinard et Tratnjek, 2016; Belghanem, 2012). Cet article témoigne d'une écriture sensible et visuelle. L'intention sensible est ainsi déployée tout au long de la recherche, jusqu'au bout du processus, de l'intention de recherche à la valorisation filmée et écrite des résultats. Ce choix d'écriture engagé et assumé défend une part d'émotion dans l'écriture scientifique et textuelle. Nous écrivons comme nous filmons, avec nos émotions. C'est aussi comme cela que nous nous sommes rencontrés et mutuellement reconnus, eux paysans agroforestiers et nous chercheur-filmeur, dans un engagement à porter d'autres pratiques, moins conventionnelles, agro-écologique en agriculture, audiovisuelle et sensible en recherche. Nous avons alors découvert dans ce travail pluridisciplinaire, par exemple que les gestes des agroforestiers, plus que les paroles, rares de surcroit, sont une manière d'écrire des émotions, leurs émotions dans l'espace géographique, leur cadre de vie, de vue et de travail, que « le travail est un rapport au corps du pays et ses gestes habitent le paysage " (Lafon, 2013). Nous avons surtout trouvé des correspondances entre les hommes, ce qu'ils sont, et le paysage qu'ils fabriquent en travaillant la terre, leur terre. Ces correspondances sont les résultats de notre recherche et le cœur de cet article qui se nourrit des pluri regards, entremêlant les regards sensibles, du géographe, de la sociologue et du cinéaste. 


\section{Écouter, regarder, comprendre}

4 Les émotions sont au cœur des deux cadres théoriques que nous mobilisons dans ce travail sociogéographique, à savoir celui de la sociologie compréhensive (Weber,1922a) et celui de la géographie des émotions, dont nous avons déjà précisé les définitions dans un article précédent (Bories, 2019).

Dans le champ de la sociologie compréhensive, nous tentons de comprendre le sens qui sous-tend les pratiques des individus et qui est donné à voir, ici les pratiques agroforestières. Nous pensons que le choix de ces pratiques est lié aux valeurs, aux "faits expériencés" par chacun, aux émotions exprimées dans les différentes interactions sociales et dans les rapports avec le métier et l'environnement. Les trajectoires et les vécus qui leurs sont associés permettent de décoder des «façons de faire» et des "façons de dire». La vision de la réalité c'est d'abord la réalité de l'individu, la représentation qu'il en a et qui renvoie à sa sensibilité, à son vivre avec ce qui l'entoure, ici, le paysage quotidien. Nous pensons que les émotions sont considérées comme des leviers de l'action (Weber, 2012). Elles sont marquées par la culture de référence et/ou la culture d'appartenance. "Chaque émotion signale ce que nous mettons de "nous-mêmes" dans l'acte de voir "l'autre" » (Hoschild, 1983). Pour nous l'autre est ce qui n'est pas moi, une autre personne, un objet, un événement, ici un paysage en lignes d'arbres.

6 Dans le champ de la géographie des émotions, nous n'étudions pas les émotions des paysans agroforestiers. Nous les acceptons, nous les enregistrons, nous les prenons en compte et nous les montrons comme des réponses affectives provoquées par un rapport à l'espace et au paysage que ces paysans fabriquent. B. Morovich et P. Desgandchamp (2016) parlent d'émotions spatialisées. Car nous croyons comme l'indique P. Guinard (2016): «que les émotions participent à notre manière de nous rapporter et de nous identifier à l'espace, de l'habiter, de nous y déplacer, de le pratiquer ou encore d'agir sur lui ». Les émotions sont alors des explications à l'action paysagère, ici agroforestière que nous étudions.

7 L'observation est centrale dans notre travail de recherche. L'observation, de surcroit quand elle est filmique, est pour nous une aventure, une pratique du terrain comme un usage du monde, un voyage qui interroge notre capacité à regarder plutôt qu'à voir, à s'arrêter ici plutôt qu'à balayer le terrain des yeux là-bas. L'observation nous engage dans une immersion attentive du pays que nous regardons. Elle n'est pas une traversée contemplative. L'observation est une exploration profonde, une expérience intime avec le paysage qui s'opère à travers l'objectif de la caméra et le micro de la perche. L'observation, ici filmique, se fait alors un examen approfondi et détaillé du terrain qui met notre attention à l'épreuve. Nous y sommes aux aguets et entièrement disposé à saisir tout ce qui se présente dans le cadre, dans le champ mais aussi hors champ, qui s'offre aux yeux comme aux oreilles. Notre observation est aussi sonore. Elle renvoie à notre faculté d'écouter plutôt qu'entendre les sons du pays dans lequel nous rentrons. Ils sont ceux de la campagne, des chants d'oiseaux au printemps ou des bourdonnements d'insectes l'été. Ils sont aussi ceux, qui s'y mélangent, du monde des Hommes, des avions ou des voitures qui filent. La caméra et le micro réduisent alors notre distance aux objets de la composition paysagère que nous filmons et à la musicalité des lieux qui s'offre à nous. La focale est celle du proche, et la lentille de l'objectif comme une fine pellicule de verre au contact de la réalité qui nous entoure. Le 
micro enregistre l'ambiance. L'observation filmique est une expérience complète, sensible et passionnée à travers laquelle nous apprenons ces lieux agroforestiers, " pour y pénétrer, s'y fondre et ne pas y demeurer visiteur » (Illouz, 2001). Le paysage devient un partenaire, pas simplement un cadre de vue qui nous accompagne. C'est une observation qui : «accorde du temps au regard pour ne pas rester à la surface des choses.» (Correa, 2020) et qui fait une place centrale à la lenteur pour décrire et s'arrêter dans les plus petits détails du pays. Nous y sommes présents, prêts à attendre, le prévu comme l'imprévu, à surprendre l'anodin. L'importance et la nature de l'observation renvoie à notre positionnement théorique sur le paysage qui n'existe qu'à la condition qu'on sache regarder et entendre les éléments qui le composent. Le paysage est alors une apparence fabriquée par la représentation (Brunet et al., 1992). Le regard et l'écoute se font un cheminement pour la pensée, comme un fil entre la matérialité et son image. L'observation ne nous renvoie pas qu'à l'espace géographique, elle nous engage aussi vers les Hommes qui fabriquent ces paysages, et vers une « rencontre sociale » (Piette, 1996) faite de conversations, de regards, "d'interactions ordinaires » qui favorisent la compréhension de l'autre (ici l'agroforestier) et de ses intentions (Fontorbes et Granié, 2013). Avec l'observation filmique nous nous mettons à l'écoute des individus et des lieux, entièrement à disposition des messages qu'ils nous envoient. Nous nous centrons sur ce qui est dit, ce qui est vu en évitant toute interprétation hâtive. Notre observation sociogéographique est compréhensive. Elle nous entraîne dans un rapport réceptif au territoire, au paysage et aux paysans agroforestiers. Cette observation-écoute prend en compte et se nourrit de toutes les émotions, les leurs mais aussi les nôtres. Nous défendons une recherche habitée par nos émotions de chercheurs. Sans ces émotions, l'interprétation paysagère serait incomplète. L'observation comporte un ressenti porté sur les traits du pays, une capacité à s'émouvoir et à s'émerveiller, un rapport intime avec les lieux, une communion entre l'homme et la terre qu'il arpente du regard. L'observation filmique permet au paysage de s'installer dans les sentiments. Les images et les sons prélevés sont empreints d'émotions. Pour nous, l'émotion permet de connaitre au plus près le monde représenté. Ce sont ces émotions que nous avons mises à l'écran pour donner à voir et à comprendre les agroforestiers et les paysages que nous avons rencontrés.

«Une fois la problématique définie, la question qui se pose au chercheur cinéaste, c'est comment montrer, comment filmer ? (...) la construction comme la pensée est toujours en mouvement. » (Fontorbes, 2020). Ainsi nous avons mis en œuvre notre poïétique qui " constitue le champ possible d'une connaissance communicable de l'art en train de se faire». (Comte, 1996). Pour en comprendre sa complétude, nous abordons le cheminement suivi. Nous restituons la manière dont nous avons produit le film recherche, les éléments qui ont présidé à cette production et la valeur scientifique et artistique qui en résulte. Nous clarifions ainsi la construction du film recherche entre les réalisateurs chercheurs et les filmés depuis le départ de la fabrication du film jusqu'à sa réception. Le processus de production dévoile les interrelations dans la rencontre, les émotions, les choix esthétiques. La rencontre avec les paysages a été saisissante et centrale dans l'écriture filmique. Les sentiments et les émotions des filmés permettent d'éclairer les rapports établis avec leur paysage et les pratiques de l'agroforesterie. «Comprendre la construction sociale de la réalité effectuée en continu par les représentations et les connaissances croisées des acteurs sociaux suppose de comprendre la manière dont se construisent socialement les émotions et les sentiments [...] qui prédéterminent les dispositions des acteurs, leurs interprétations à l'égard de 
cette réalité, leur attention à l'égard du monde - de - la vie. (Farrugia, 2016). C'est la confiance partagée et le goût de la réciprocité avec les agroforestiers qui a permis de construire le film entre/de l'homme et son paysage.

\section{Planter des arbres}

9 Nous n'avons pas découvert dans cette recherche qu'il existe une motivation paysagère dans l'engagement agroforestier ( $C f$. étude exploratoire). Les agroforestiers sont des paysans réceptifs au paysage (Bories et Rue, 2017) qui reversent cette sensibilité dans l'action, le métier et sa pratique. En revanche nous avons découvert dans le paysage les traits et les caractères de cette sensibilité. Nous avons donc appris par la forme du paysage ce qu'il nous raconte des liens sensibles qui unissent l'homme à la pratique agroforestière. Et nous avons découvert grâce au paysage que l'agroforesterie va bien au-delà d'une technique agro-écologique dont toutes les études vantent les performances agronomiques, écologiques et économiques pour déclencher l'engagement, sinon la promouvoir. Notre recherche et la méthodologie spécifique mise en place révèlent autre chose de l'agroforesterie qu'un lot de bénéfices mesurables et de valeurs marchandes. C'est une occasion d'écrire avec des arbres dans le pays un rapport au monde, au territoire, à la nature, à l'agriculture et au métier. C'est une manière de positionner l'idée dans l'espace géographique, de placer dans le paysage des valeurs, des convictions, des besoins, des aspirations et des émotions, peut-être même des rêves.

«Il y a un côté agréable, stimulant de remettre des arbres là où il n'y en avait plus. Ça fait toujours quelque chose. Couper un gros arbre ça apporte toujours une tristesse, ce n'est pas une expérience facile. Et là ça fait l'effet inverse, on rapporte quelque chose, on vient réimplanter des arbres alors qu'ils avaient été arrachés avant. Ça fait du bien, ça fait plaisir $»^{4}$

Le paysage agroforestier nous a appris des correspondances entre l'homme, ce qu'il est et ce qu'il fait qui méritent ici d'être analysées.

\section{Dans le paysage}

11 Le paysage de l'agroforesterie est un paysage d'interstices. Il se glisse en timbre-poste comme un détail dans les étendues, principalement céréalières, de notre campagne d'étude. Dissous dans une mosaïque agricole composée de parcelles étalées et de grands labours. Le paysage agroforestier est un paysage de bout de terre, de quelques hectares à peine, rarement plus d'une dizaine. Au départ c'est un paysage de l'essai, de la tentative qui renvoie à une particularité de profils dans le goût des agroforestiers pour l'expérimentation. Il se construit par à-coups, jamais en grand format pour les paysans agroforestiers que nous avons rencontrés ${ }^{5}$. C'est un paysage des petites proportions, pas celui "du clinquant" et de l'immense. Un morceau de terrain-là, attenant à la ferme, puis l'année suivante c'est un autre que l'on plante, un peu plus loin. La composition agroforestière se dessine comme ça, par bout de parcelles, dans une fragmentation de plantation, à l'envie, lente et progressive des terres aux alentours de l'exploitation. Les compositions agroforestières que nous avons étudiées ne sont jamais brutales, même si la figure nous interpelle par la rangée, la rectitude et la verticalité des alignements d'arbres dans une campagne arasée (et interpelle aussi certains agroforestiers) : 
«Le seul hic, vu notre intervention on a fait des alignements bien droits. A un moment donné on y était un petit peu obligé...C'est le seul problème. La ligne droite dans la nature n'existe pas. En soi ce n'est pas très grave, ça fait quelque chose de cartésien, d'aligné. C'est ce que je regrette le plus. Parce que en fait moi dans ma façon de penser je n'aime pas ce qui est trop droit... $»^{6}$ soutiennent. Le paysage de l'agroforesterie s'appuie, la plupart du temps, sur l'inclinaison des terres à stabiliser (mais pas toujours), ou s'insinue dans un plus vieux maillage de haies bocagères, pour le renforcer. Ce paysage s'imprime avec discrétion, douceur et légèreté dans le tissu agricole et nous renvoie comme cela à l'idée de l'interstice : recoin géographique. C'est un paysage qui ne se remarque pas. Il se déniche et il surprend, un peu par hasard, dans les détours, les intervalles et les marges d'une campagne " dégradée » par les incitations à l'arrachage des années 70 et uniformisée par les grands parcellaires. Le paysage agroforestier se fait une particularité "silencieuse" dans un ensemble agraire plus vaste et le reflet d'une pratique alternative qui se glisse elle aussi dans les interstices d'un modèle agricole encourageant à l'étalement des champs.

Le paysage de l'agroforesterie met dans l'image du lieu cette première correspondance entre un positionnement géographique et un positionnement professionnel. Il organise dans la parcelle une rencontre entre l'idéologie qui porte l'élan d'un autre système agricole, certainement d'une résistance, et la figure "esthétique" qui la met en mouvement dans le paysage. L'agroforesterie établit une correspondance entre le paysage, la pratique et l'homme par l'idée du recoin, de la marge géographique et de la "marginalité" (dans le sens de l'alternative) d'une pratique et de ceux qui s'essayent à la développer. Ces agroforestiers sont d'ailleurs paysans, pas agriculteurs: «Je suis paysan agroforestier $»^{7}$ (C'est par cette " affirmation d'un soi-même » que l'un d'entre eux commence le film recherche). Dans le récit les agroforestiers nous font part des modalités pratiques de leur quotidien. Le nommer pour se raconter, raconter le nous est analysé comme un élément explicatif de leur identité individuelle et collective (Ricoeur, 1983-1985) L'agroforesterie, comme nous l'avons observée, se fait la représentation concrète et visible dans le paysage de cette différence, de pratiques et de façon de penser.

\section{Dans le geste}

La relation entre le paysage agroforestier, le paysan et sa pratique ne se dit pas. Elle s'observe, comme le paysage et ce que nous avons fait avec la caméra tout au long de cette recherche. Les agroforestiers ne nous parlent pas de paysage dans le sens du discours et d'une verbalisation paysagère comme nous l'avions écrit (Bories et Rue, 2017). Ils sont discrets comme les recoins géographiques dans lesquels ils agissent. Ce sont des paysans silencieux, économes de la parole, de surcroît quand elle est paysagère et qu'elle renvoie à une intimité plus difficile à partager. Le paysage est une intimité. La plupart des agroforestiers rencontrés sont des paysans réservés :

«Sur la symbolique, planter un arbre c'est quand même fort. Mais ça c'est des choses qui ne se disent pas trop. Le monde paysan est quand même très pudique làdessus. Des fois il n'y a pas besoin de grands mots, juste un regard, des gestes, une attitude...ça peut très bien passer comme ça aussi. $»^{8}$ 
15 Le paysage agroforestier se fait alors le reflet dans le cadre du pays de l'agroforestier lui-même. Les agroforestiers préfèrent la construction de la trace à l'expression par le verbe, la réalité du paysage à sa description. Ils fabriquent dans le paysage ce qu'ils sont eux même. Alors le paysage est eux et eux sont ce paysage ; c'est ce que nous avons voulu montrer en images et en sons. C'est par le travail et surtout par le geste agroforestier qu'ils nous racontent qui ils sont et ce qu'ils sont dans ce qu'ils font. Les gestes agroforestiers sont des paroles, leurs paroles paysagères. Elles nous parlent d'agroforesterie dans le paysage, directement au contact de la terre. Ces paroles sont totalement incarnées et chargées de sens parce qu'elles sont situées « au bon endroit » c'est à dire dans la parcelle agroforestière.

Le geste agroforestier est évidemment un geste technique. Les agroforestiers sont d'ailleurs des techniciens très pointus et de fins connaisseurs en agronomie, en écologie des milieux ou en biologie végétale. Leur geste est bien plus qu'une mobilisation de connaissances et qu'une mise en application "froide" et désincarnée de méthodes. Le geste agroforestier que nous avons observé et filmé est habité par la sensibilité de celui qui l'exécute, dans l'application à tracer des lignes de plantation bien droites, dans la précaution à bien praliner les racines du jeune plant avant de le mettre en terre, dans la générosité du paillage, dans le regard expert et affectueux avant de tailler et dans la caresse sur l'écorce après l'avoir fait : « allez ça suffit comme ça... »". Le geste technique reverse dans le paysage une "attention", "trahit » une proximité avec la nature et se fait ainsi "plus épais" qu'une exécution maîtrisée de savoirs faires techniques. Le geste n'est pas qu'un moyen, une démonstration de légitimité professionnelle. Il est bien plus que cela. Il est une attention, la marque d'une affection, la trace du soin. Alors nous l'avons filmé comme cela et pour cela, en rentrant dans l'infime détails, en s'approchant au plus près de la main qui presse le sécateur, des bras qui mélangent le pralin ou actionnent la perche à élaguer, en s'invitant par la confiance au plus près de l'intime des corps qui travaillent l'arbre, et parfois de la voix chuchoteuse qui lui parle. Le geste est une articulation entre le corps et l'esprit, une mise en mouvement qui donne à voir dans le paysage comment s'opèrent deux grandes catégories de rapports, entre l'homme et le temps d'abord, entre l'homme et la nature ensuite.

\section{Dans un autre rapport avec le temps}

17 Le geste agroforestier nous parle dans le paysage d'un autre rapport au temps. Avec le temps de la tâche à réaliser d'abord. Elle se répète autant de fois qu'il y a de lignes et d'arbres dans la parcelle. Le geste nous parle de cadence dans le paysage ou le paysage nous raconte une cadence de geste. Ce n'est pas la cadence infernale des temps modernes de Chaplin. C'est la cadence du temps à prendre, qui n'est pas un temps compté "pour faire vite" et mesuré pour faire "beaucoup" mais un temps nécessaire "pour faire bien", pas nécessairement d'ailleurs dans les règles d'un art agroforestier en construction. Le temps à prendre est le temps qu'il faut pour que l'agroforestier soit satisfait de ce qu'il a réalisé, pour que le résultat lui convienne sans forcément qu'il soit chiffré. La satisfaction ne se mesure pas. Elle se ressent et s'apprécie. Les lignes se tracent au cordeau et les protections anti gibier s'enfilent à la main sur les tuteurs. La tâche est, la plupart du temps, manuelle. C'est la main qui fait le lien avec une agriculture moins mécanisée, plus ancienne et traditionnelle, pas moins moderne, si la modernité n'est pas réduite à l'apparat technique et à la mécanique de précision. La 
paysannerie est une forme de modernité. Et ici c'est avec la main comme matériel de travail que le geste agroforestier remet symboliquement le temps paysan dans le paysage. La tâche agroforestière nous parle dans la parcelle et dans le cadre de vue "du temps d'avant". Le geste nous parle aussi d'un autre rapport au temps dans le paysage par la surveillance. Ce temps renvoie à celui de l'observation et à une déambulation, qui se fait à pied, pas en tracteur, dans ou aux abords de la parcelle. Cette visite "de contrôle" n'est pas forcément programmée. Elle est "hors temps" et se fait, comme ça, par opportunité "d'un temps libre" dans la journée, de façon régulière et elle s'étire tout au long de l'année, le matin, le midi ou le soir, pendant plusieurs années. C'est le temps du coup d'œil "dans le temps qui passe" pour s'assurer qu'il n'y a pas de problème, de tuteur arraché par le gibier par exemple, pour se rassurer avec des arbres qui se développent correctement. Et pour se faire plaisir. Le temps de la surveillance est un temps pour se mettre "hors du temps", un peu comme ces paysans se mettent « hors champ » et pour retrouver une forme de lenteur à travers le paysage qu'ils fabriquent, doucement.

Tous les gestes agroforestiers impriment un rythme différent dans le cadre de travail qui se remarque dans le cadre de vue. Les gestes et leur paysage nous parlent du temps qui passe autrement et qui se passe autrement, à distance de la rapidité, de la mesure et de l'évaluation pour valider une efficacité de production. Il y a dans les gestes et les paysages agroforestiers la présence d'un temps qui s'étire, du temps certes compté aussi pour eux mais jamais perdu, du temps long qui s'émancipe des logiques de courtterme dominant les marchés agricoles et du temps long qui renvoie symboliquement au temps de l'arbre. En prenant le temps de l'arbre comme rythme de travail, les agroforestiers nous parlent de patience dans le paysage. Ils nous racontent à leur façon l'histoire actuelle des urgences climatiques ou sanitaires et avec l'agroforesterie nous présentent une manière de se faire résilient, en choisissant de prendre le temps. Les agroforestiers nous parlent avec les gestes dans le paysage d'un ralentissement nécessaire pour produire, pour exister et vivre autrement :

«On est dans un système où il faut de plus en plus courir, après l'argent, après le travail. Ce n'est pas positif. Il faut se calmer. On ne peut pas observer en courant. Il faut s'asseoir et observer. Pour écouter, pour sentir, pour vivre. Et quand je me tourne et que je vois ce que je vois là, je vis » ${ }^{10}$

19 Avec les gestes ces agroforestiers nous parlent d'un engagement à situer leur travail dans une autre temporalité. Le paysage se fait l'image dans le pays d'un changement de cadence, moins effrénée et l'expression d'une préconisation, comme un conseil à se montrer plus raisonnable.

\section{Dans un autre rapport avec la nature}

20 Le geste agroforestier est dans le paysage l'expression de rapprochements avec la nature, l'occasion d'un contact avec la parcelle, les arbres, et le lieu. Le rapprochement est concret, se fait au toucher, à l'œil et à l'écoute, tout en marchant dans les lignes pour exécuter la tâche ou surveiller la plantation. Ce rapprochement s'opère sur le temps du travail, mais aussi tôt le matin avant que la journée débute ou plus tard le soir quand elle se finit :

«En buvant le café le matin, depuis la cuisine j'ai un accès direct et visuel sur la parcelle. Ça me permet de la surveiller et de me rendre compte que ce travail est 
chouette, que c'est valorisant. Si je ne vais pas au quotidien sur la parcelle, en tout cas depuis ici, au quotidien, je la vois! $»^{11}$

21 C'est un rapprochement toujours furtif, presque un geste volé, à l'abri des regards, un acte intime. C'est une caresse au feuillage de l'arbre à tailler, quelques mots qui lui sont adressé, une contemplation silencieuse au fond du champ, autant de petites attentions qui nous en disent beaucoup sur un besoin tactile et sensoriel avec la terre et les éléments, presque spirituel parfois. Le geste est alors une présence à la terre, une façon d'être là près de quelque chose qui semble être plus haut que soi.

«Vous imaginez une parcelle vide semée ou pas semée et vous visualisez une agroforesterie où tout fleurit. Et là, il n'y a plus la monotonie d'une parcelle vide. Quand tout est en fleur, tout est arboré, tout pousse, tout fleurit...et ça donne la pêche. C'est la première expression qui me vient, ça me file la pêche ça! C'est viscéral. Ça ne s'explique pas trop. Il n'y a pas trop de mots pour ça. Surtout au printemps quand tout se réveille. Et là c'est vibratoire en fait, c'est électromagnétique. On sent cette énergie, le champ, la chaleur...Tu te sens bien, tu peux t'asseoir au pied d'un arbre. Le quart d'heure il passe très vite. C'est un capteur de tristesse, voilà un arbre. Vous vous asseyez au pied, il vous prend la mélancolie et il vous recharge de positif. $»^{12}$

C'est une marque d'affection pour et dans le paysage agroforestier, une émotion qui se met en mouvement par le corps et qui se coule dans l'élégance de la discrétion de ces hommes planteurs. Le geste nous dévoile dans le paysage agroforestier non seulement un besoin de contact avec la matière mais aussi un lien très profond et une proximité affective avec le vivant.

Au-delà du contact il y a l'idée d'un partenariat avec la nature. Le rapprochement est aussi idéel, allant cette fois du corps à l'esprit, du geste à l'idée. Le geste marque une complicité avec et dans le paysage. Ce rapprochement nous parle alors de pratique agricole. Il nous dit qu'il ne s'agit pas de faire SUR mais de faire AVEC la nature, autrement dit de concevoir le travail agricole non pas dans la domination, la maîtrise de la nature et de l'ensemble des processus naturels qui régissent la production agricole, mais comme un travail partenarial avec les entités naturelles. Le geste est une manière d'installer la co-production entre les hommes et la nature dans le paysage, de construire la collaboration entre l'agroforestier et sa terre, de faire de la terre une partenaire et de se replacer à niveau pour «s'atteler à une véritable écologie des relations entre humains et non-humains » (Larrère, 2015).

\section{Planter la caméra}

Travailler avec l'audiovisuel dans le cadre de cette recherche a consisté à montrer les liens entre l'homme et son paysage, dans le sens où l'homme habite le paysage, le construit et en contrepoint le paysage habite l'homme et façonne ici l'identité professionnelle et personnelle de l'agroforestier.

Nous avons choisi de faire prendre une photographie qui représente son paysage par chacun des agroforestiers. La photographie est le résultat d'une interaction entre le sujet, ici l'agroforestier et l'objet, ici le paysage :

«En prenant cette photo, je me remémore ce qu'était ce site il y a de nombreuses années...Il y avait particulièrement une haie qui traversait et qui allait rejoindre le bois qui était là-bas, que je revois... En plus j'ai participé à éliminer cette haie à l'époque, je m'en souviens...Et puis je me souviens de tous les animaux que nous 
avions et qui venaient pâturer dans toute cette zone...Et puis ça me remémore qu'en

2016 on a fait la première plantation...et on l'a faite sur cette zone-là. ${ }^{13}$ percevoir et de représenter l'autre " (Conord, 2007). La photographie contribue à la connaissance, nous l'utilisons comme valeur de réalité, la réalité du sujet. La prise de vue permet d'une part de montrer par l'image le lien intime que chacun établit avec son paysage et d'autre part, de recueillir les récits qui justifient ce point de vue. Leur choix spontané du cadre associe les agroforestiers à l'écriture audiovisuelle. Cette manière de procéder s'inscrit dans l'expression des sentiments contre la réserve. Ce faisant, la rencontre avec les agroforestiers rentre dans une histoire, notre histoire commune en train se faire. Comme dit Fabien Truong (2015) « nous jouons la carte de la monstration ». De fait les agroforestiers reconstruisent une deuxième fois un morceau de leur paysage par la photographie.

avons placé les agroforestiers dans leur paysage, d'abord en plan fixe, enracinés comme un arbre. Ainsi ils inscrivent leur signature dans le paysage. Le regard fixe renvoie à la lenteur du temps, à l'immobilité, à l'observation et au temps qui s'éternise. Ces paysans ne parlent pas, on voit seulement leurs cheveux qui «bougent» sous le souffle du vent, comme bougent les feuillages dans le paysage. « Depuis ses débuts, par les plans les plus simples, le cinéma nous a souvent rappelé que ces formes élémentaires d'émerveillement ne sont pas mortes : feuilles qui bougent, en silence, à l'arrière-plan du Déjeuner de Bébé des frères Lumière, ramures d'acacias dans L'Eclipse d'Antonioni, feuillage du cognassier dans Le songe de la lumière blanche d'oliviers dans Au travers des oliviers (...), nombreux sont les plans où par des instants de participation, le regard du cinéma a cherché à saisir l'arbre, à exprimer des forces à l'œuvre dans ses formes.. " (Mottet, 2002). Ce choix de mise en scène montre la force du cinéma pour nous faire rentrer dans les émotions et les correspondances qui s'établissent ici entre l'homme et le paysage.

Les voix des agroforestiers sont en off. Elles nous racontent des extraits de trajectoires, de transmissions, de rapports à la nature, aux paysages. Ces paroles d'agroforestiers que nous entendons alors qu'ils ont le regard presque figé, accroché au paysage, à leur paysage, nous impressionnent en ce sens où elles nous invitent à rentrer dans leur pensée. Nous avons voulu que l'image des agroforestiers reflète leur pensée et que leurs paroles soient inscrites dans leur pensée. Ce choix de mise en scène met le spectateur en situation de rencontre intime avec les agroforestiers et place le paysage sur le devant de la scène. C'est alors que le paysage devient personnage (Bories, 2019). Nous proposons ici une autre manière de filmer la parole et le paysage. "Ce qui est mis en activité dans cette expérience de terrain c'est pour moi l'approche des contextes par la production de sens endogène. C'est à dire produit par les individus eux-mêmes. Oui il m'importe de recueillir la subjectivité ». (Fontorbes, 2003). Nous avons recueilli le rapport intime entre ces agroforestiers et leur paysage; autrement dit le sens qu'ils donnent à leur métier, à leur travail, à leur environnement, à leur vie. «Elle (l'image) porte, en effet, quelque part en elle, explicitement ou non, les marques du regard dont elle est l'aboutissement. En d'autres termes, l'image est un "montré » qui porte en lui le point de vue particulier de celui qui l'a créé ». (Terrenoire, 1985). Les chercheurs filmeurs et les agroforestiers sont dans une relation audiovisuelle maitrisée et scientifiquement pertinente. Chacun sait que ce qui est produit s'adresse à des destinataires. C'est bien dans la complicité filmeurs-filmés que nous avons pu recueillir

EchoGéo, 56 | 2021 
les regards, les mots, les sons, les gestes et ainsi construire le propos scientifique que nous proposons à partir du sens commun donné par les agroforestiers.

L'utilisation du drone nous a permis, au-delà d'une vision d'ensemble du paysage de chaque agroforestier, d'en faire un portrait en miroir avec le portrait du paysan agroforestier (cf. écriture filmique), de découvrir les recoins, les interstices que nous avons évoqués plus haut. On a pu ainsi voir et comprendre l'attachement de ces paysans au moindre petit détail de leur parcelle, de leur rangée. Le drone nous a permis une description visuelle précise. Chaque détail filmé fait avancer la compréhension. Le drone a participé à l'activité d'observation. « La notion d'observation renvoie d'ailleurs le plus souvent non pas à la seule activité du regard mais non seulement à l'ensemble des techniques utilisées pour comprendre les actions des hommes (...) mais aussi à toute posture scientifique en général ». (Piette, 1996). Le drone nous a placé au cœur de l'activité agroforestière. Le détail renvoie à toute la complexité du choix de cette manière de faire de l'agriculture et/ou de l'élevage.

La longueur des plans dans ce film recherche nous place et nous installe dans le temps long de l'activité agroforestière; Le rythme du film est calqué sur le rythme de la nature, du temps de l'arbre. Nous montrons qu'il y a une adéquation entre la réalité montrée, la réalité pratiquée et la réalité vécue. Ce film est là " pour nous apprendre quelque chose que nous ne connaissions pas auparavant » sur ce monde, comme dirait Howard S Becker (2010). Le métier d'agroforestier est décliné entre « être » et « faire ». C'est cela que montre le film: une vie de travail dans, avec et pour la nature. En quelque sorte un métier qui se pratique à deux dans un respect mutuel des rythmes biologiques. L'homme et l'arbre, une vie aurait pu être le titre du film car c'est cela que nous tentons d'expliquer par notre travail filmique en ne cantonnant pas l'esthétique à la seule interprétation, et en donnant une place importante à l'émotion. Le tournage s'est effectué dans une continuité narrative maitrisée mais non rigide puisque nous avons pris en compte ce qui se présentait à nous dans l'instant et dans la relation qui se construisait avec les filmés.

\section{«Ce film c'est vraiment nous »}

Notre posture méthodologique se situe entre engagement et mise à distance. Avec le dispositif filmique, notre regard et notre écoute constamment en éveils, nous nous impliquons dans la situation d'interactions avec les filmés mais nous restons attentifs aux effets qui font partie de cette réalité. Nous organisons en reflet à l'écran les correspondances que nous découvrons dans l'analyse. Comme le dit Edgar Morin «nous sommes des observateurs - sujets" (Morin, 1994). La réalité filmée est un produit de cette situation sociale. Nous n'évacuons jamais ce que produit le regard camera. Cela suppose une reconnaissance réciproque des chercheurs filmeurs et des filmés sans laquelle la recherche avec les images et les sons ne peut se faire. "La reconnaissance et le don sont liés et c'est à cette condition que nous avons pu faire le film. La promesse du don conclut la reconnaissance (Fontorbes et Granié, 2011). Le film n'a pu se réaliser que parce que les agroforestiers ont "donné leurs paroles et leur faire » et en retour les chercheurs filmeurs leur donnent le film. Quelle vérité scientifique et humaine lorsque à la fin de la projection du film recherche, lorsque la lumière s'allume, nous entendons un filmé s'exclamer: "Ce film c'est vraiment nous $»^{14}$. Ce travail de recherche en images et en sons nous a profondément plongé dans l'intimité des hommes et des lieux 
dont ils s'occupent. Il nous a enrichis scientifiquement par ce que nous y avons trouvé de beauté, de sensibilité et de nuances, beaucoup appris derrière la caméra des recoins où se cachent des paysages en lignes d'arbres et où œuvrent des paysans discrets. Et si nous sortons de cette recherche complètement convaincu de la part paysagère dans l'action agroforestière, ce travail nous interroge plus que jamais sur l'urgence. L'urgence est sociale, environnementale, climatique, économique. Mais l'urgence est aussi paysagère dans ces campagnes qui continuent de s'aplanir. Ainsi nous comprenons qu'il y a dans l'engagement agroforestier des paysans que nous avons rencontré plus encore qu'une conscience paysagère, le souci d'un cadre de vue, et peutêtre l'intention d'utiliser la lenteur de l'arbre pour faire face à l'urgence paysagère dans leur campagne. Cela comme un paradoxe qui consiste à prendre le temps pour aller vite. Dans les lignes d'arbres les agroforestiers se construisent un chemin de pensée durable et de pratiques agroécologiques.

L'enjeu scientifique et pédagogique est central dans nos travaux filmiques. "Transmettre de la connaissance avec le film participe à la construction des savoirs, par la mobilisation d'éléments contextués extrêmement divers tels que des situations sociales, culturelles, interactionnelles, montrées, dites, vécues; des émotions exprimées, des gestes, des intonations produites, des lieux, des paysages donnés » (Granié, 2005).

\section{BIBLIOGRAPHIE}

Belghanem A., 2012. Stephen Kalberg, Les valeurs, les idées et les intérêts. Introduction à la sociologie de Max Weber. Questions de communication, $\mathrm{n}^{\circ} 21$, p. 344-347.

Bories O., 2019. Faire du paysage un "personnage". Les atouts de la méthode filmique dans la production d'images paysagères. Revue française des méthodes visuelles [En ligne], $\mathrm{n}^{\circ} 3$. URL : rfmv.fr/numeros/3/articles/4-faire-du-paysage-un-personnage/

Bories O, Rue M., 2017. Quand des agriculteurs agroforestiers haut-garonnais nous parlent d'arbre et de paysage. Projets de paysage : revue scientifique sur la conception et l'aménagement de l'espace [En ligne], n 16. URL: http://journals.openedition.org/paysage/5049 - DOI: https:// doi.org/10.4000/paysage.5049

Bories O., 2008. Le paysage dans les manières d'habiter des résidents du périurbain : le cas de l'agglomération toulousaine. Thèse Géographie-aménagement, Université Toulouse 2 Le Mirail.

Brunet R. et al.,1992. Les mots de la géographie, dictionnaire critique. Paris, Reclus, La Documentation Française, $470 \mathrm{p}$.

Comte R., 1996. La poïétique de l'artiste philosophe Paul Valéry. Revue de la société internationale de poḯtique, $n^{\circ} 5$, Dossier Paul Valéry.

Conord S., 2007. Usages et fonctions de la photographie. In Arrêt sur Images, Photographie et anthropologie. Ethnologie Française, PUF, Introduction.

Correa P., 2020. Matins clairs. Ed. L'Iconoclaste, 66 p. 
Farrugia F., 2016. Psychanalyse des bons et des mauvais sentiments. Un syndrome narratif collectif. In Ferreol (dir.), Sentiments et Émotions. Proximités-Sociologie, Bruxelles, EME.

Fontorbes J.-P., 2020. L'audiovisuel, une écriture scientifique. In Pouzenc M., Charlery de la Masselière B. (dir.), Étudier les ruralités contemporaines. Toulouse, PUM, p. 161-177.

Fontorbes J.-P., Granié A.-M., 2013. Traces de l'objet, traces du sujet. Comment les pratiques des traces contribuent à la compréhension de l'identité socioprofessionnelle du chercheur. Sciences de la société [En ligne], n 89. URL: http://journals.openedition.org/sds/283 - DOI: https:// doi.org/10.4000/sds.283

Fontorbes J.-P., Granié A.-M., 2011. L'auto récit, la mise en scène de l'identité narrative au cinéma. Cahiers Louis Lumière, $\mathrm{n}^{\circ} 8$, p. 65-72.

Fontorbes J.-P., 2003. Le corps du regard. Thèse de doctorat communication et audiovisuel, UT2J, tome 1, 7 films-recherche, $238 \mathrm{p}$.

Granié A.-M., 2005. Figures de Constructions Identitaires. Regards Croisés. Le film, le réalisateur et la sociologue. HDR Tome 2, UTM, UMR Dynamiques Rurales.

Guinard P., Tratnjek B., 2016. Géographies, géographes et émotions. Carnets de géographes [En ligne], $n^{\circ}$ 9. URL: http://journals.openedition.org/cdg/605 - DOI: https://doi.org/10.4000/cdg.605

Hoschild A. R., 1983 [2017, traduction française]. Le prix des sentiments. Au cœur du travail émotionnel. Paris, La Découverte, 312 p.

Illouz T., 2001. L'ombre allongée. Ed. Fayard, 129 p.

Lafon M.-H., 2013. Traversée. Ed. Créaphis, 33 p.

Larrère C., Larrère R., 2015. La nature pour partenaire. CNRS Le journal.

Morovich B, Desgandchamp P., 2016. Créations sonores et émotions : une géographie strasbourgeoise. Carnets de géographes [En ligne], n 9. URL: http://journals.openedition.org/cdg/ 689 - DOI: https://doi.org/10.4000/cdg.689

Morin E., 1994. Extrait de Sociologie. Repris dans La Complexité Humaine. Champs Flammarion, p. 302-303.

Mottet J., 2002. Avant-propos. In Mottet J. (dir.), L'arbre dans le paysage. Pays/Paysage, Champ Vallon, $280 \mathrm{p}$.

Piette A., 1996. Ethnographie de l'action. Paris, Métaillé, 203 p.

Ricoeur P., 1983-1985. Temps et Récit. Paris Seuil, 3 tomes.

Becker H. S., 20098. Comment parler de la société. Artistes, écrivains, chercheurs et représentations sociales, Trad. de l'anglais par Christine Merllié-Youn. Paris, La Découverte, $318 \mathrm{p}$.

Terrenoire J.-P., 1985. Image et sciences sociales : l'objet et l'outil. Revue Française de Sociologie, vol. 26, n 3, p. 509-527.

Truong F., 2015. Le Goût des autres : sociologie des intentions et intentions sociologiques. L'année sociologique, vol. $65, \mathrm{n}^{\circ} 1$, p. 125-147.

Weber M., 2012. Sociologie des émotions. Revue du MAUSS, vol. 40 n 2, p. 285-299.

Weber M., 1971 [1ère éd. 1922]. Économie et société. Paris Plon. 


\section{NOTES}

1. Si le « paysan agroforestier » est un idéal-type dominant sur notre terrain, nous pouvons peutêtre supposer qu'il existe aussi d'autres profils, plutôt agriculteurs agroforestiers, moins ancrés dans cette conception paysanne de l'agroforesterie. Nous n'en avons pas rencontré dans ce travail et nous n'en parlons donc pas.

2. En 2014 nous avons conduit une étude exploratoire auprès de 15 agroforestiers haut-garonnais et en partenariat avec l'association Arbres et Paysages d'Autan visant à mesurer l'inclinaison paysagère dans leur engagement. Nous formulions déjà l'hypothèse d'un lien entre l'action agroforestière et le projet de paysage.

3. Le film-recherche À l'ombre des champs, 2020 (Réal. Bories O. \& Fontorbes J.-P.) est un travail de recherche pluridisciplinaire et collectif auquel ont participé Elsa Pibou (MCF contractuelle en sociologie, ENSFEA, LISST Dynamiques rurales), Jean-Michel Cazenave (Chargé de projet audiovisuel, ENSFEA, LISST Dynamiques rurales) et Anne-Marie Granié (comité scientifique). Ensemble nous faisons partie du collectif écriture filmique de l'ENSFEA-Dynamiques Rurales.

4. Paroles de paysans agroforestiers.

5. Peut-être existe-t-il ailleurs de plus grandes parcelles.

6. Paroles de paysans agroforestiers.

7. Paroles de paysans agroforestiers.

8. Paroles de paysans agroforestiers.

9. Paroles de paysans agroforestiers.

10. Paroles de paysans agroforestiers.

11. Paroles de paysans agroforestiers.

12. Paroles de paysans agroforestiers.

13. Paroles de paysans agroforestiers.

14. Paroles de paysans agroforestiers

\section{RÉSUMÉS}

L'agroforesterie rhabille des campagnes dénudées par l'étalement parcellaire. Elle remet des lignes d'arbres dans le pays et joue de ses effets de rangs, de contours et de détourages pour créer la surprise dans le paysage. Nous sommes rentrés avec la caméra dans ces champs rayés d'arbres. Pendant une année nous y avons rencontré et filmé des paysans agroforestiers. Cinq. A Auriacsur-Vendinelle, Caubiac et Fontenilles en Haute-Garonne, Noilhan et Auch dans le Gers. Cet article présente les résultats de notre recherche filmique et met en mots nos images et nos sons. Il nous parle d'émotions, de paysage agroforestier, et de ces Hommes qui écrivent discrètement dans le territoire ce qu'ils sont, en plantant des arbres dans leurs parcelles. Nous avons filmé leurs gestes comme une parole agroforestière silencieuse. Nous avons découvert que dans ces lignes d'arbres, les agroforestiers se construisent un chemin de pensée durable et de pratiques agro-écologiques, un rapport avec le monde.

Agroforestry rehabilitates countryside denuded by the spread of plots. It puts lines of trees back into the country and plays with its effects of rows, contours and outlines to create surprise in the landscape. We went with the camera into these tree-striped fields. For a year we met and filmed agroforestry farmers. Five. In Auriac-sur-Vendinelle, Caubiac and Fontenilles in Haute-Garonne, 
Noilhan and Auch in the Gers. This article presents the results of our filmic research and puts our images and sounds into words. It talks about emotions, agroforestry landscapes, and about the people who discreetly write who they are in the territory by planting trees in their plots. We filmed their gestures as a silent agroforestry word. We discovered that in these lines of trees, the agroforesters are building a path of sustainable thought and agro-ecological practices, a relationship with the world.

\section{INDEX}

Thèmes : Sur l'Image

Mots-clés : agroforesterie, paysage, socio-géographie des émotions, film-recherche

Keywords : Agroforestry, Landscape, Socio-geography of emotions, Film-research

\section{AUTEURS}

\section{OLIVIER BORIES}

Olivier Bories, olivier.bories@ensfea.fr, est maître de conférences et réalisateur à l'ENSFEA, LisstDynamiques rurales. Il a récemment publié :

- Eychenne C., Bories O., Noûs C., 2020. (Éco)pâturage, (éco)pastoralisme : la gestion de l'espace par les troupeaux, éléments d'analyse et de compréhension. Carnets de géographes [En ligne], $\mathrm{n}^{\circ}$ 14. URL: http://journals.openedition.org/cdg/6086 - DOI: https://doi.org/10.4000/cdg.6086 - Bories O., 2019. Faire du paysage un "personnage". Les atouts de la méthode filmique dans la production d'images paysagères ", Revue française des méthodes visuelles [En ligne], 3. Revue française des méthodes visuelles [En ligne], $\mathrm{n}^{\circ} 3$. URL : rfmv.fr/numeros/3/articles/4-faire-dupaysage-un-personnage/

- Bories O., Eychenne C., Leterrier G., Dubreuilh J.-L., 2019. Quand les moutons circulent sur les trottoirs de nos villes. Urbanités [En ligne], n 11. URL: https://www.revue-urbanites.fr/11bories-et-al/

\section{JEAN-PASCAL FONTORBES}

Jean-Pascal Fontorbes, jean-pascal.fontorbes@ensfea.fr, est maitre de conférences HDR et réalisateur à l'ENSFEA, membre du Lisst-Dynamiques rurales. Il a récemment publié :

- Fontorbes J.P., 2020. L'audiovisuel, une écriture scientifique. In Pouzenc M., Charlery de la Masselière B.(dir.), Étudier les ruralité contemporaines. Toulouse, PUM, p. 161-174.

- Fontorbes J.P., Granié A.M., 2019. Nos films recherche. Histoires de rencontres, « cadrages débordements. Revue Française des Méthodes Visuelles [En ligne], n³. URL: https://rfmv.fr/ numeros/3/articles/11-nos-films-recherche-histoires-de-rencontres/

- Fontorbes J.P., 2016. Création cinématographique et Recherche en SHS. Avant-Propos. Entrelacs, hors-série $n^{\circ} 2$, p. 5-9.

\section{ANNE-MARIE GRANIÉ}

Anne-Marie Granié, annemarie.granie@orange.fr, est Professeur émérite du Lisst-Dynamiques rurales. Elle a récemment publié :

- Bories O., Fontorbes J.P., Granié A.M., 2018. Quand l'agriculture prend de la hauteur. Filmer au jardin potager sur le toit de la clinique Pasteur à Toulouse. Vertigo. La revue électronique en sciences de l'environnement [En ligne], Hors-série n 31. URL: http://journals.openedition.org/ 
vertigo/21398 - DOI: https://doi.org/10.4000/vertigo.21398

- Fontorbes J.P., Granié A.M., 2017. Paysans au quotidien, le quotidien des paysans, rapports aux temps. À partir du film Paysans on y croit dur comme ferme. In Sebag J., Durand J.-P., Louveau C., Queirolo Palmas L., Stagi L. (dir.), Sociologie visuelle et filmique. Le point de vue de la vie quotidienne, Genova University Press, p 36-46.

- Fontorbes J.P, Granié A.M., 2016. Herencia, une errance sociologique et artistique. Entrelacs, hors-série $\mathrm{n}^{\circ} 2$, p. 149-160. 